\title{
Tipificaciones en la familia Lauraceae de la Flora del Paraguay
}

\author{
Lorenzo Ramella \& Gabrielle Barriera
}

\begin{abstract}
RAMELLA, L. \& G. BARRIERA (2018). Typifications in the Lauraceae family of the Flora of Paraguay. Candollea 73: 109-113 In Spanish, English and Spanish abstracts. DOI: http://dx.doi.org/10.15553/c2018v731a11

The typification of 15 names described in the Lauraceae family from Paraguayan collections in the genera Aiouea Aubl., Nectandra Rottb., Ocotea Aubl., Oreodaphne Nees \& Mart. and Phoebe Nees is presented. Lectotypes are designated for: Nectandra lanceolata var. paraguariensis Hassl., Ocotea confusa Hassl., Ocotea gracilipes Mez, Ocotea lanceolata f. latifolia Hassl., Ocotea suaveolens var. robusta Hassl. and Oreodaphne suaveolens Meisn. A second step lectotypification is made for Nectandra megaphylla Hassl.
\end{abstract}

\section{Resumen}

RAMELLA, L. \& G. BARRIERA (2018). Tipificaciones en la familia Lauraceae de la Flora del Paraguay. Candollea 73: 109-113. En español, resúmenes en inglés y español. DOI: http://dx.doi.org/10.15553/c2018v731a11

Se tipifican 15 nombres descritos en la familia Lauraceae a partir de colecciones de Paraguay en los géneros Aiouea Aubl., Nectandra Rottb., Ocotea Aubl., Oreodaphne Nees \& Mart. y Phoebe Nees. Seis lectótipos son designados: Nectandra lanceolata var. paraguariensis Hassl., Ocotea confusa Hassl., Ocotea gracilipes Mez, Ocotea lanceolata f. latifolia Hassl., Ocotea suaveolens var. robusta Hassl. y Oreodaphne suaveolens Meisn. Una lectotipificación de segundo paso se realiza para Nectandra megaphylla Hassl.

\section{Keywords}

LAURACEAE - Aiouea - Nectandra - Ocotea - Oreodaphne - Phoebe - Paraguay - Typification

\footnotetext{
Dirección de los autores:
}

Conservatoire et Jardin botaniques de la Ville de Genève, C.P. 71, 1292 Chambésy, Suiza. E-mail: florapara@ville-ge.ch 


\section{Introducción}

La publicación de la familia Lauraceae de la Flora del Paraguay (BERNARDI, en preparación) hace necesario la tipificación de todos los nombres descritos a partir de colecciones de Paraguay en esta familia. Para tal propósito, identificamos el material original de cada uno de estos nombres. Designamos 6 lectótipos y en un caso realizamos una lectotipificación de segundo paso. Los nombres aceptados en la Flora se han indicado usando negritas. Se encontraran las consideraciones taxonómicas utilizadas para la redacción de esta familia en una nota separada (BERNARDI, 2018).

\section{Tipificaciones}

Aiouea hassleri Mez in Bull. Herb. Boissier ser. 2, 3: 229.1903.

Holotypus: Paraguay. Canindeyú: "In campo Ipé hú", X.1898-1899, Hassler, E. 5004 (G [G00229914]!; iso-: G [G00228277, G00228278]!).

= Aiouea trinervis Meisn. in A. DC., Prodr. 15(1): 83. 1864.

Nectandra briquetii Hassl. in Annuaire Conserv. Jard. Bot. Genève 21: 93. 1919.

Holotypus: Paraguay. Amambay: "In altaplanitie Sierra de Amambay. Orilla mont. Limeira", VI.1912-1913, Hassler, E. 11247 (G [G00228405]!; iso-: A, BM, F, G [G00228406, G00228407, G00228408]!, K, MPU, P).

Notas. - Se indica en van Der Werff et al. (2015: 49) la necesidad de lectotipificar entre los pliegos de la colección Hassler 11247 conservados en G. El espécimen [G00228405] es claramente la colección original en la cual figuran tanto los elementos diagnósticos como un análisis ilustrado de las partes florales de la mano del autor. Se trata por lo tanto del holótipo y una lectotipificación es aquí superflua.

Nectandra dubia Hassl. in Annuaire Conserv. Jard. Bot. Genève 21: 97. 1919.

Holotypus: Paraguay. Amambay: "In altaplanitie Sierra de Amambay”, X.1912-1913, Hassler, E. 11984 (G [G00228438]!).

$=$ Ocotea catharinensis Mez in Bot. Jahrb. Syst. 30, Beibl. 67: 19. 1901.
Nectandra lanceolata var. grandifolia Mez in Bull. Herb. Boissier ser. 2, 3: 794. 1903.

Lectotypus (primer paso: RoHwer, 1993: 225; segundo paso: van der Werff et al., 2015: 47): Paraguay. San Pedro: "In silva pr. fl. Corrientes", IX.1898-1899, Hassler, E. 4484 (G [G00228372]!; isolecto-: BM [BM000947267] foto!, F [V0061510F] foto!, G [G00228371, G00228373, G00228374]!, GH [GH00041944, GH00041945] foto!, K [K000576200] foto!, NY [NY00355460] foto!, P [P00711031, P00711032] foto!). Syntypus: ParaguaY. Canindeyú: "In dumeto pr. Igatimi", X.1898-1899, Hassler, E. 4876 (G [G00228378]!; isosyn-: BM [BM000092851] foto!, G [G00228375, G00228376, G00228377]!, K [K000576201] foto!, NY [NY00355461] foto!, P [P00711029] foto!).

= Nectandra lanceolata Nees \& Mart. in Linnaea 8: 47. 1833.

Nectandra lanceolata var. paraguariensis Hassl. in Annuaire Conserv. Jard. Bot. Genève 21: 92. 1919.

Lectotypus (in hoc loco designatus): Paraguay. Canindeyú: "In dumeto pr. Igatimi", X.1898-1899, Hassler, E. 4876 (G [G00228378]!; isolecto-: BM [BM000092851] foto!, G [G00228375, G00228376, G00228377]!, K [K000576201] foto!, NY [NY00355461] foto!, P [P00711029] foto!). Syntypus: Paraguay. San Pedro: "In silva pr. fl. Corrientes", IX.1898-1899, Hassler, E. 4484 (G [G00228372]!; isosyn-: BM [BM000947267] foto!, F [V0061510F] foto!, G [G00228371, G00228373, G00228374]!, GH [GH00041944, GH00041945] foto!, K [K000576200] foto!, NY [NY00355460] foto!, P [P00711031, P00711032] foto!).

= Nectandra lanceolata Nees \& Mart. in Linnaea 8: 47. 1833.

Notas. - Dos síntipos (Hassler 4484 y Hassler 4876) fueron utilizados para la descripción de $N$. lanceolata var. grandifolia Mez (1903) y N. lanceolata var. paraguariensis Hassl. (1919). La lectotipificación de la var. grandifolia en base a la colección Hasler 4484 permite utilizar el segundo síntipo disponible (Hassler 4876) como lectótipo de la var. paraguariensis. Esta última variedad no es por lo tanto ilegítima contrariamente a lo indicado por RoHWer (1993: 225).

Nectandra lanceolata var. parvifolia Mez in Bull. Herb. Boissier ser. 2, 3: 794. 1903.

Holotypus: Paraguay. Canindeyú: "In silva pr. Curuguaty", IX.1898-1899, Hassler, E. 4590 (G [G00228368]!; iso-: BM, G [G00228367, G00228369, G00228370]!, K, L, NY, P).

$=$ Nectandra lanceolata Nees \& Mart. in Linnaea 8: 47.1833. 
Notas. - El holótipo de N. lanceolata var. parvifolia Mez es idéntico a la lectotipificación superflua efectuada por vaN DER WerfF et al. (2015: 47). La descripción original indica claramente una única colección (Hassler 4590) y en $\mathrm{G}$ se puede perfectamente determinar la colecta [G00228368] que conforma el material original con las características de la diagnosis.

Nectandra megaphylla Hassl. in Annuaire Conserv. Jard. Bot. Genève 21: 92. 1919.

Lectotypus (primer paso: RoHWER, 1993: 196; segundo paso: in hoc loco designatus): Paraguay. Amambay: "Sierra de Amambay in via silvatica Picada Esperanza", VIII.1907-1908, Hassler, E. E T. Rojas 10584 (G [G00228418]!; isolecto-: A [A00041951] foto!, B [B100185040] foto!, BM [BM000092844] foto!, F [F0061511F] foto!, G [G00228416, G00228417]!, K [K000642330] foto!, NY [NY00355472] foto!, P [P00711038, P00711039, P00711040] foto!, S [S-R-7220] foto!). Syntypus: ParaguaY. Amambay: "In altaplanitie Sierra de Amambay, entre R. Dorado y Sto. Tomas", VIII.1912-1913, Hassler, E. 11295 (G [G00228421]!; isosyn-: BM, F, G [G00228419, G00228420]!, GH, K, $\mathrm{NY}, \mathrm{P})$.

Notas. - Rohwer (1993: 196) designó la colección Hassler E Rojas 10584 como lectótipo, pero sin especificar el espécimen original. Lo designamos formalmente aquí.

Nectandra membranacea f. floribunda Hassl. in Annuaire Conserv. Jard. Bot. Genève 21: 96. 1919.

Holotypus: ParaguaY. Amambay: "Sierra de Amambay ad margines humidas silvarum pr. Esperanza", VII.19071908, Hassler, E. \& T. Rojas 10529 (G [G00228280]!; iso-: G [G00228291, G00228292, G00228864]!, MO, NY).

$=$ Nectandra angustifolia (Schrad.) Nees \& Mart. in Linnaea 8: 48.1833.

Notas. - Se indica en van der Werff et al. (2015: 49) la necesidad de lectotipificar entre los pliegos de la colección Hassler \& Rojas 10529 conservados en G. El espécimen [G00228280] es claramente la colección original en la cual figura un análisis ilustrado de las partes florales de la mano del autor. Se trata por lo tanto del holótipo y una lectotipificación es aquí superflua.
Ocotea confusa Hassl. in Annuaire Conserv. Jard. Bot. Genève 21: 82. 1919.

Lectotypus (in hoc loco designatus): Paraguay. Amambay: "Sierra de Amambay in campis altis arenosis Cerro Cora", VI.1908, Hassler, E. E T. Rojas 10477 (G [G00228490]!; isolecto-: BM [BM000092796] foto!, G [G00228487, G00228488, G00228489]!, K [K000602328] foto!, MO [MO247559] foto!, NY [NY00355588] foto!, P [P00756988, P00756989, P00756990] foto!, US [US00048574] foto!). Syntypi: Paraguay. San Pedro: "In campo Apépú (Tapiraguay)", VIII.1898-1899, Hassler, E. 4362 (G [G00228497]!; isosyn-: A, F, G [G00228496, G00228498, G00229969]!, K, MO, NY, P, S); “In campo Apépú (Tapiraguay)", VIII.1898-1899, Hassler, E. $4362 a$ (G [G00228500]!; isosyn-: G [G00228499, G00228501, G00229968]!, K). Alto Paraná: "In campo pr. fl. Capibary”, XII.1898-1899, Hassler, E. 5900 (G [G00228492]!). Canindeyú: "In campo Ipé hú Sierra Maracayu”, X.18981899, Hassler, E. 4941 (G [G00228494]!; isosyn-: G [G00228493, G00228495, G00229967]!, K, NY, P).

= Ocotea densiflora (Meisn.) Mez in Jahrb. Königl. Bot. Gart. Berlin 5: 301. 1889.

Notas. - El espécimen Hassler \& Rojas 10477 [G00228490] es el único síntipo que cuenta con una diagnosis detallada de la mano de Hassler, razón por la cual lo elegimos como lectótipo.

Ocotea gracilipes Mez in Repert. Spec. Nov. Regni Veg. 3: 71. 1906.

$\equiv$ Ocotea lanceolata var. gracilipes (Mez) Hassl. in Annuaire Conserv. Jard. Bot. Genève 21: 85. 1919.

Lectotypus (in hoc loco designatus): Paraguay. Caaguazú: "In viciniis Caaguazú", III.1905, Hassler, E. 9165a (B [B100185324] foto!; isolecto-: BM [BM000092861] foto!, G [G00228565, G00228566, G00228567]!, K [K000352470] foto!, NY [NY00355609] foto!, P [P00756905] foto!). Syntypus: Paraguay. Caaguazú: "In viciniis Caaguazú", III.1905, Hassler, E. 9165 (B [B100185325] foto!; isosyn-: A, BM, G [G00228560, G00228561, G00228562, G00228563, G00228564]!, K, MPU, NY, P).

Notas. - De acuerdo al protólogo, el material original se encuentra en el herbario de Berlín (B). 
Ocotea lanceolata f. latifolia Hassl. in Annuaire Conserv. Jard. Bot. Genève 21: 85. 1919.

Lectotypus (in hoc loco designatus): Paraguay. Amambay: "In altaplanitie et declivibus Sierra de Amambay", XI.1907-1908, Hassler, E. E T. Rojas 10834 (G [G00228594]!; isolecto-: B [B100185296] foto!, MPU [MPU018857] foto!, P [P00756815, P00756816] foto!). Syntypus: ParaguaY. Amambay: "Sierra de Amambay silvulas formans in arenosis pr. Cerro Corá", VI.1907-1908, Hassler, E. E T. Rojas 10476 (G [G00228590]!; isosyn-: B [B100185298] foto!, G [G00228587, G00228588, G00228589]!, K [K000352468] foto!).

= Ocotea floribunda (Sw.) Mez in Jahrb. Königl. Bot. Gart. Berlin 5: 325.1889.

Notas. - La colección Hassler \& Rojas 10834 designada aquí como lectótipo, presenta las características descritas para la f. Latifolia en el protólogo de Hassler: "foliis lanceolatis cum ovato-ellipticis in eodem specimine, 85/25-95/45 mm", y esto de manera general en todos los especímenes conservados en $\mathrm{G}, \mathrm{B}$, MPU y P. El material original, parte de la colección personal de Hassler conservada en G, lleva una nota del autor en la etiqueta: "Ocotea lanceolata (Nees) forma fol. latioribus". Agrega: "A Mez surreptam", lo que indicaría que se trata de un ejemplar parcial, el resto habiendo quedado en las manos de Mez. El otro síntipo, Hassler $\Xi^{2}$ Rojas 10476, presenta hojas lanceolados sin la presencia en el mismo ejemplar de hojas ovadas-elípticas con las proporciones indicadas en el protólogo. Esta característica es compartida entre todos los síntipos consultados (B, G, K).

Ocotea pseudocorymbosa Hassl. in Annuaire Conserv. Jard. Bot. Genève 21: 87. 1919.

Holotypus: Paraguay. Amambay: "In altaplanitie Sierra de Amambay. Nuvera”, XI.1912-1913, Hassler, E. 11416 (G [G00228503]!; iso-: A, BM, F, G [G00228502, G00228504]!, MPU, NY, P).

= Ocotea diospyrifolia (Meisn.) Mez in Jahrb. König1. Bot. Gart. Berlin 5: 374. 1889.

Notas. - El holótipo Hassler 11416 es considerado por van DER WerfF et al. (2015: 51) como síntipo lo cual es erróneo, puesto que la descripción original menciona una sola colecta como tipo. El hecho de tener varios especímenes en $\mathrm{G}$ de esta misma colección no permite considerarlos como síntipos, puesto que se puede fácilmente identificar el material original [G00228503] por contar con una descripción diagnóstica y el análisis floral ilustrado de la mano del autor.
Ocotea suaveolens var. robusta Hassl. in Annuaire Conserv. Jard. Bot. Genève 21: 90. 1919.

Lectotypus (in hoc loco designatus): Paraguay. Cordillera: "Paraguaria centralis: in regione lacus Ypacaray. S. Bernardino", X.1913, Hassler, E. 12310 (G [G00228898]!; isolecto-: B [B100185427] foto!, F [V0061660F] foto!, G [G00228897]!, US [00997597] foto!). Syntypi: ParaGUAY. Cordillera: "Itacurubí-Unión”, VI.1898, Endlich, $R$. 239 (G [G00228854]!); "In silvis virgineis Cordillera de Altos”, X.1895, Hassler, E. 1342 (G [G00228873]!; isosyn-: G [G00228872, G00228874]!); "Ad marginem paludis pr. San Bernardino”, X.1885-1895, Hassler, E. 1367 (G [G00228875]!; isosyn-: G [G00228876, G00228877]!); "Paraguaria centralis: in regione lacus Ypacaray. Pie Cordilleras", VII.1913, Hassler, E. 11803 (G [G00228893]!; isosyn-: B, F, G [G00228894]!, US). Guairá: "Itangu, près de Villa-Rica, dans les bois”, 14.X.1874, Balansa, B. 2016 (G [G00228848]!; isosyn-: G [G00228847, G00228849]!). Paraguarí: "In silvis montanis pr. Cordillera de Paraguary", IX.1895, Hassler, E. 1156 (G [G00228869]!; isosyn-: G [G00228868, G00228870]!, K); "In silvis montanis pr. Cordillera de Paraguary", IX.1895, Hassler, E. $1156 a$ (G [G00228871]!). Central: "L'Assomption, dans les bosquets", IV.1874, Balansa, B. 2022 (G [G00228852]!; isosyn-: G [G00228850, G00228851]!). Amambay: "In altaplanitie Sierra de Amambay", VII.1912-1913, Hassler, E. 11653 (G [G00228892]!). Bolivia: Rusby, H. H. 2672 (G [G00369447]!; isosyn-: US).

$=$ Ocotea suaveolens (Meisn.) Hieron. in Bol. Acad. Nac. Ci. Republ. Argent. 4: 437. 1881.

Notas. - La colección Hassler 12310 [G00228898] designada aquí como lectótipo además de llevar las características de la diagnosis original, lleva la anotación "Typus 2010. 1803 etcl" la cual refiere a una numeración alternativa de este mismo ejemplar figurando en el espécimen, así como también del especímen de la colección Hassler 11803 [G00228893]. Esta nota va acompañada de un análisis ilustrado de la flor y estambre por el propio Hassler.

Oreodaphne suaveolens Meisn. in A. DC., Prodr. 15(1): 136. 1864.

$\equiv$ Ocotea suaveolens (Meisn.) Hieron. in Bol. Acad. Nac. Ci. Republ. Argent. 4: 437.1881.

Lectotypus (in hoc loco designatus): Paraguay. Central: "Assomption" [f.], VI.1858, Gibert, E. J. 56 (K [K000442013] foto!; isolecto-: NY [NY00355784] foto!, P [P00711095] foto!). Syntypi: ParaguaY. Central: "Assomption"[fl. gem.], VI.1858, Gibert, E. J. 74 (K [K000442033] foto!). Sin indicación del departamento: "Gd. Chaco" [sterilis], VI.1858, Gibert, E. J. 21 (K [K000442032] foto!; isosyn-: NY [NY00355785] foto!). 
Notas. - De acuerdo al protólogo, el material original se encuentra en el herbario Hooker, ahora en K. De los 3 síntipos elegimos a la colección Gibert 56, la única que está en flor.

Phoebe paraguariensis Hassl. in Annuaire Conserv. Jard. Bot. Genève 21: 79. 1919.

$\equiv$ Cinnamomum paraguariense (Hassl.) Kosterm. in Reinwardtia 6: 22.1961.

Holotypus: Paraguay. Amambay: "In altaplanitie Sierra de Amambay. Montes húmedos Sto. Tomas", VIII.19121913, Hassler, E. 11305 (G [G00228251]!; iso-: A, BM, F, G [G00228252, G00228253, G00228254]!, K, MPU, NY, P, S).

= Cinnamomum triplinerve (Ruiz \& Pav.) Kosterm. in Reinwardtia 6: 24. 1961.

Notas. - El holótipo de P. paraguariensis Hassl. es idéntico a la lectotipificación superflua efectuada por RoHde et al. (2017: 1103). La descripción original indica claramente una única colección (Hassler 11305) y en G se puede perfectamente determinar la colecta [G00228251] que conforma el material original por contar con una descripción diagnóstica y el análisis floral ilustrado de la mano del autor.

\section{Referencias}

Bernardi, L. (2018). Novedades taxonómicas en Nectandra Rottb. y Ocotea Aubl. (Lauraceae) de la Flora del Paraguay. Candollea 73: 105-108.

Bernardi, L. (en preparación). Lauraceae. Fl. Paraguay 50.

Rohde, R., B. Rudolph, K. Ruthe, F.G. Lorea-Hernández, P.L. Rodrigues de Moraes, J. Li \& J.G. Rohwer (2017). Neither Phoebe nor Cinnamomum - the tetrasporangiate species of Aiouea (Lauraceae). Taxon 66: 1085-1111.

Rohwer, J.G. (1993). Lauraceae: Nectandra. Fl. Neotrop. Monogr. 60. van der Werff, H., C.A. Zanotti \& J.C. Ospina (2015). Lauraceae. Fl. Argentina 15: 41-57. 